\title{
Complexity Results for the Spanning Tree Congestion Problem
}

Yota Otachi

Hans L. Bodlaender

Erik Jan van Leeuwen

Technical Report UU-CS-2010-007

March 2010

Department of Information and Computing Sciences

Utrecht University, Utrecht, The Netherlands

www.cs.uu.nl 
ISSN: 0924-3275

Department of Information and Computing Sciences Utrecht University P.O. Box 80.089 3508 TB Utrecht The Netherlands 


\title{
Complexity Results for the Spanning Tree Congestion Problem
}

\author{
Yota Otachi* $^{*}$ Hans L. Bodlaender ${ }^{\dagger} \quad$ Erik Jan van Leeuwen ${ }^{\ddagger}$
}

\begin{abstract}
We study the problem of determining the spanning tree congestion of a graph. We present some sharp contrasts in the complexity of this problem. First, we show that for every fixed $k$ and $d$ the problem to determine whether a given graph has spanning tree congestion at most $k$ can be solved in linear time for graphs of degree at most $d$. In contrast, if we allow only one vertex of unbounded degree, the problem immediately becomes NP-complete for any fixed $k \geq 10$. For very small values of $k$ however, the problem becomes polynomially solvable. We also show that it is NP-hard to approximate the spanning tree congestion within a factor better than $11 / 10$. On planar graphs, we prove the problem is NP-hard in general, but solvable in linear time for fixed $k$.
\end{abstract}

\section{Introduction}

Spanning tree congestion is a relatively new graph parameter, which was formally defined by Ostrovskii [21] in 2004. Prior to Ostrovskii [21], Simonson [25] studied the same parameter under a different name to approximate the cutwidth of outerplanar graphs. Although several graph theoretical results have been presented [6, 16, 17, 18, 20, 22] after Ostrovskii [21], so far, no results on the complexity of the problem were known. In this paper, we present the first such results. The parameter is defined as follows. Let $G$ be a graph and $T$ a spanning tree of $G$. The detour for an edge $\{u, v\} \in E(G)$ is the unique $u-v$ path in $T$. We define the congestion of $e \in E(T)$, denoted by $c n g_{G, T}(e)$, as the number of detours that contain $e$. The congestion of $G$ in $T$, denoted by $c n g_{G}(T)$, is the maximum congestion over all edges in $T$. The spanning tree congestion of $G$, denoted by $\operatorname{stc}(G)$, is the minimum congestion over all spanning trees of $G$. We denote by STC the problem of determining whether a given graph has spanning tree congestion at most given $k$. If $k$ is fixed, we denote the problem by $k$-STC.

${ }^{*}$ Department of Computer Science, Gunma University, 1-5-1 Tenjin-cho, Kiryu, Gunma 376-8515, Japan. JSPS Research Fellow. otachi@comp.cs.gunma-u.ac.jp

${ }^{\dagger}$ Institute of Information and Computing Sciences, Utrecht University, P.O. Box 80.089, 3508 TB Utrecht, The Netherlands. hansb@cs.uu.nl

\#Department of Informatics, University of Bergen, P.O. Box 7803, 5020 Bergen, Norway. E.J.van.Leeuwen@ii.uib.no 
The name of the parameter comes from the following analogy [6]: Edges of $G$ are roads, and edges of $T$ are those roads which are cleaned from snow after snowstorms. For an edge $h \in E(T)$, it is natural to define the congestion of $h$ as the number of detours passing through $h$. Clearly, the congestion of the busiest roads should be minimized. The tree spanner problem [5] is a variant of the problem, which minimize the dilation, that is, the length of the longest detours. Several pairs of congestion and dilation problems are known [23]. The most famous pair is the cutwidth problem and the bandwidth problem.

The rest of the paper is organized as follows. Section 2 provides some definitions and basic facts. In Section 3, we study the problem for planar graphs, and show that STC for planar graphs is NP-complete, and $k$-STC for planar graphs is solvable in linear time. In Section 4, we show that $k$-STC can be solved in linear time for $1 \leq k \leq 3$. In Section 5, we show that $k$-STC can be solved in linear time also for graphs of bounded degree. In Section 6 , we show that $k$-STC is NP-complete for edge weighted graphs if $k \geq 10$. Using the result of Section 6, we show in Section 7 that for $k \geq 10, k$-STC is NP-complete for simple unweighted graphs with only one vertex of unbounded degree. In the last section, we conclude the paper and show the approximation hardness of the spanning tree congestion.

\section{Preliminaries}

We extend the notion of spanning tree congestion to edge weighted graphs, by defining the congestion of an edge as the sum of the weights of edges whose detours pass through the edge. We denote by $w(F)$ the sum of weights of edges in $F$ for an edge set $F \subseteq E(G)$.

Let $G$ be a connected graph. For $S \subseteq V(G)$, we denote by $G[S]$ the subgraph induced by $S$. For an edge $e \in E(G)$, we denote by $G-e$ the graph obtained by the deletion of $e$ from $G$. For $A, B \subseteq V(G)$, we define $E_{G}(A, B)=\{u, v \in E(G) \mid u \in A, v \in B\}$. For $S \subseteq V(G)$, we define the boundary edges of $S$, denoted by $\theta_{G}(S)$, as $\theta_{G}(S)=E_{G}(S, V(G) \backslash S)$. Using this notation, we can redefine $c n g_{G, T}(e)$ as $c n g_{G, T}(e)=\left|\theta_{G}\left(A_{e}\right)\right|$, where $A_{e}$ is the vertex set of one of the two components of $T-e$. From this redefinition through boundary edges, we can see that $c$-cut trees defined by Fekete and Kremer [11] and spanning trees of congestion at most $c$ are equivalent.

For an edge $e$ in a tree $T$, we say that $e$ separates $A$ and $B$ if $A \subseteq A_{e}$ and $B \subseteq B_{e}$, where $A_{e}$ and $B_{e}$ are the vertex sets of the two components of $T-e$. Clearly, if $T$ is a spanning tree of $G$ and $e \in E(T)$ separates $A$ and $B$, then cng $_{G, T}(e) \geq|E(A, B)|$ (if $G$ is weighted, $c n g_{G, T}(e) \geq w(E(A, B))$ ). If $e$ separates $A$ and $B$, we also say that $e$ divides $A \cup B$ into $A$ and $B$.

From the definition of the spanning tree congestion, the following proposition holds.

Proposition 2.1. The spanning tree congestion of $G$ equals the maximum spanning tree congestion of its biconnected components.

Ostrovskii [21] showed the following lower bound on the spanning tree congestion of graphs.

Lemma 2.2 ([21]). Let $G$ be a graph, $u, v \in V(G)$. If $G$ has $k$ edge disjoint $u-v$ paths, then $\operatorname{stc}(G) \geq k$. 
Let $G$ be a graph. We say that a graph $H$ is obtained from $G$ by an edge subdivision if $V(H)=$ $V(G) \cup\{w\}$ and $E(H)=E(G) \backslash\{\{u, v\}\} \cup\{\{u, w\},\{w, v\}\}$ for some edge $\{u, v\} \in E(G)$ and a new vertex $w$. We say that $H$ is a subdivision of $G$ if $H$ can be obtained from $G$ by a finite sequence of edge subdivisions. If $H$ is a subdivision of a subgraph of $G$, then $H$ is a topological minor of $G$.

The concept of treewidth was introduced by Robertson and Seymour in their project of Graph Minor Theory (see [24] for example). A tree decomposition of a graph $G$ is a pair $(\mathcal{X}, T)$, where $T$ is a tree and $\mathcal{X}=\left\{X_{i} \mid i \in V(T)\right\}$ is a collection of subsets of $V(G)$ such that

- $\bigcup_{i \in V(T)} X_{i}=V(G)$,

- for each edge $\{u, v\} \in E(G)$, there is a node $i \in V(T)$ such that $u, v \in X_{i}$, and

- for each $v \in V(G)$, the set of nodes $\left\{i \mid v \in X_{i}\right\}$ forms a subtree of $T$.

The elements in $\mathcal{X}$ are called bags. The width of a tree decomposition $(\mathcal{X}, T)$ equals $\max _{i \in V(T)}\left|X_{i}\right|-$ 1. The treewidth of $G$, denoted by $t w(G)$, is the minimum width over all tree decompositions of $G$.

\section{Spanning tree congestion of planar graphs}

Ostrovskii [22] has asked whether STC can be solved in polynomial time for planar graphs. By combining a number of known results, we answer this question negatively (assuming $P \neq N P$ ), and show that $k$-STC can be solved in linear time for planar graphs. Our results follow easily from some known results for the tree spanner problem. Let $G$ be a graph and $T$ a spanning tree of $G$. If $\operatorname{dist}_{T}(u, v) \leq k$ for any $\{u, v\} \in E(G)$, then $T$ is a tree $k$-spanner [5]. We denote by $t s p(G)$ the minimum number $k$ such that $G$ has a tree $k$-spanner. For planar graphs, the following results are known.

Lemma 3.1 ([11]). It is NP-complete to decide $t s p(G) \leq k$ for planar graphs $G$ and integers $k$.

Lemma 3.2 ([10]). For every fixed $k, \operatorname{tsp}(G) \leq k$ can be decided in linear time for planar graphs $G$.

A dual graph $G^{*}$ of a planar graph $G$ is a graph that has the vertex set $\mathcal{F}(G)$, the faces of a certain embedding of $G$, and in which two vertices $f, f^{\prime} \in \mathcal{F}(G)$ are adjacent in $G^{*}$ if and only if the two faces $f$ and $f^{\prime}$ have a common edge in $G$. It is known that a graph $G$ is planar if and only if $G$ is a dual graph of a planar graph (see e.g. [9]). Since a cut in $G$ corresponds to a cycle in $G^{*}$, the following relation holds.

Lemma 3.3 ([11]). For any planar graph $G, \operatorname{stc}(G)=\operatorname{tsp}\left(G^{*}\right)+1$.

A planar embedding of a planar graph can be constructed in linear time by an algorithm proposed by Hopcroft and Tarjan [15]. From a planar embedding of a planar graph $G$, we can easily construct geometrically a dual graph $G^{*}$ (see e.g. [19]). Thus, from Lemma 3.3, we can have the conclusions of this section.

Theorem 3.4. It is NP-complete to decide $\operatorname{stc}(G) \leq k$ for planar graphs $G$ and integers $k$.

Theorem 3.5. For every fixed $k$, stc $(G) \leq k$ can be decided in linear time for planar graphs $G$. 


\section{Linear time solvability of $k$-STC for $1 \leq k \leq 3$}

In this section, we show that $k$-STC can be solved in linear time for $1 \leq k \leq 3$. First, we give characterizations for graphs of spanning tree congestion one and two.

Theorem 4.1. For a connected graph $G$, $\operatorname{stc}(G)=1$ if and only if $G$ is a tree.

Proof. If $G$ be a tree, then clearly $\operatorname{stc}(G)=1$. Assume $G$ has a cycle $C$. Then, for any two vertices in $C, G$ has two edge disjoint paths between them. Thus, by Lemma 2.2, $G$ cannot have any cycle.

A graph $G$ is a cactus graph if no two cycles in $G$ have a common edge.

Theorem 4.2. For a connected graph $G, \operatorname{stc}(G)=2$ if and only if $G$ is not a tree but a cactus graph.

Proof. Clearly, every biconnected component of a cactus graph $G$ is either a cycle or a single edge, and thus, $G$ has spanning tree congestion at most two. It is easy to verify that a biconnected graph $G$ has no vertex pair $u, v$ such that $G$ contains three edge disjoint $u-v$ paths if and only if $G$ is either a cycle or a single edge. Thus, from Proposition 2.1 and Lemma 2.2, the theorem holds.

Obviously, the recognition of trees and cactus graphs can be done in linear time, by using standard depth first search techniques (see e.g. [7]). For $k=3$, we need the following lemma.

Lemma 4.3. For a graph $G$, if $\operatorname{stc}(G) \leq 3$, then $G$ is planar.

Proof. Suppose $\operatorname{stc}(G) \leq 3$ and $G$ is not planar. From Kuratowski's Theorem (see e.g. [9]), $G$ has either $K_{5}$ or $K_{3,3}$ as a topological minor. If $G$ has $K_{5}$ as a topological minor, then clearly $G$ contains two vertices such that $G$ has at least four edge disjoint paths between them. From Lemma 2.2, we have $\operatorname{stc}(G) \geq 4$, which is a contradiction. Thus, $G$ contains $K_{3,3}$ as a topological minor. Let $G^{\prime}$ be this topological minor, and $X=\left\{x_{1}, x_{2}, x_{3}\right\}, Y=\left\{y_{1}, y_{2}, y_{3}\right\} \subset V\left(G^{\prime}\right)$ be the two sets corresponding to the two color classes of $K_{3,3}$. By Lemma 7.2 edge subdivisions do not change the spanning tree congestion. Thus, $\operatorname{stc}\left(G^{\prime}\right)=\operatorname{stc}\left(K_{3,3}\right)$. Moreover, by Hruska's result that shows $\operatorname{stc}\left(K_{m, n}\right)=m+n-2$ [16], we can conclude $\operatorname{stc}\left(G^{\prime}\right)=4$. Now we need the following two propositions.

Proposition 4.4. Let $H$ be a connected graph and $H^{\prime}$ a connected subgraph of $H$. If a spanning tree $S$ of $H$ has a spanning tree $S^{\prime}$ of $H^{\prime}$ as a subgraph, then $\mathrm{cng}_{H}(S) \geq \mathrm{cng}_{H^{\prime}}\left(S^{\prime}\right)$.

Proof. Let $e \in E\left(S^{\prime}\right) \subseteq E(S)$. Assume $e$ divides $V(H)$ into $A$ and $B$, and $V\left(H^{\prime}\right)$ into $A^{\prime}$ and $B^{\prime}$. Clearly, $A^{\prime} \subseteq A$ and $B^{\prime} \subseteq B$. Thus, cng $_{H, S}(e)=|E(A, B)| \geq\left|E\left(A^{\prime}, B^{\prime}\right)\right|=\operatorname{cng}_{H^{\prime}, S^{\prime}}(e)$.

Proposition 4.5. Let $H$ be a connected graph, $S$ a spanning tree of $H$, and $A, B \subset V(H)$. If $H$ has $p$ edge disjoint paths $P_{1}, \ldots, P_{p}$ between $A$ and $B$, and $e \in E(S)$ separates $A$ and $B$, then cng $_{H, S}(e) \geq p$. Moreover, if e does not belong any $P_{i}$, then $\operatorname{cng}_{H, S}(e) \geq p+1$. 
Proof. For each $P_{i}$, there exists at least one edge $e_{i}$ such that the detour of $e_{i}$ in $S$ passes through the edge $e$. Since the paths $P_{1}, \ldots, P_{p}$ are edge disjoint, $c n g_{H, S}(e) \geq p$. Since $e$ itself is the detour for $e, \operatorname{cng}_{H, S}(e) \geq p+1$ if $e \notin\left\{e_{i} \mid 1 \leq i \leq p\right\}$.

We will show that $c n g_{G, T}(e)>3$ for any spanning tree $T$ of $G$. If $T$ has a spanning tree of $G^{\prime}$ as a subgraph, then from Proposition $4.4 \mathrm{cng}_{G}(T) \geq 4$. This implies that $T$ contains a path $P$ between two vertices of $X \cup Y$ such that $P$ contains an edge $e$ not in $E\left(G^{\prime}\right)$. Edge $e$ divides $X \cup Y$ into two nonempty sets $A$ and $B$. It is easy to see that for any nonempty partition $(A, B)$ of $X \cup Y$, there exist at least three edge disjoint paths between $A$ and $B$. Thus, $c n g_{G, T}(e) \geq 4$ from Proposition 4.5.

From Theorem 3.5 and Lemma 4.3, 3-STC can be solved in linear time, with the linear time algorithm for recognizing planar graphs [15]. This proves the following theorem.

Theorem 4.6. For $1 \leq k \leq 3, k$-STC can be solved in linear time.

\section{Linear time solvability of $k$-STC for graphs of bounded de- gree}

In this section, we show that $k$-STC can be solved in linear time for graphs of bounded degree. To this end, we use Courcelle's theorem and a connection between the spanning tree congestion and the treewidth. Courcelle [8] showed that every problem expressible in $\mathrm{MS}_{2}$ can be solved in linear time for graphs of bounded treewidth, where $\mathrm{MS}_{2}$ is a graph logic in the monadic second-order logic (see also [14]). In $\mathrm{MS}_{2}$, we are allowed to use the incident relation inc, the membership relation $\in$, and variables over vertices, edges, vertex sets, and edge sets.

Theorem 5.1. For graphs of bounded treewidth, $k$-STC can be solved in linear time.

Proof. We show that $k$-STC is expressible in $\mathrm{MS}_{2}$. Let $G=(V, E)$ and $|G|_{2}:=\langle V, E$, inc $\rangle$. For a vertex $v \in V$ and an edge $e \in E$, inc $(v, e)$ if and only if $e$ has $v$ as an endpoint. For $F \subseteq E(G)$, we denote by $G\langle F\rangle$ the subgraph induced by $F$, that is, $E(G\langle F\rangle)=F$ and $V(G\langle F\rangle)=\bigcup_{\{u, v\} \in F}\{u, v\}$.

We first define the following basic expressions:

$$
\begin{aligned}
\operatorname{Deg} 1\left(v_{1}, E_{1}\right) & :=\left(\exists e_{1} \in E_{1}\right)\left(\forall e_{2} \in E_{1}\right)\left(e_{1}=e_{2} \Longleftrightarrow \operatorname{inc}\left(v_{1}, e_{2}\right)\right), \\
\operatorname{Part}\left(V_{1}, V_{2}, V_{3}\right) & :=V_{2} \neq \emptyset \wedge V_{3} \neq \emptyset \wedge\left(V_{2} \cup V_{3}=V_{1}\right) \wedge\left(V_{2} \cap V_{3}=\emptyset\right), \\
\operatorname{Adj}\left(v_{1}, v_{2}, E_{1}\right) & :=v_{1} \neq v_{2} \wedge\left(\exists e_{1} \in E_{1}\right)\left(\operatorname{inc}\left(v_{1}, e\right) \wedge \operatorname{inc}\left(v_{2}, e\right)\right), \\
E_{1}=\operatorname{Ind}\left(V_{1}\right) & :=\left(\forall e_{1}\right)\left(e_{1} \in E_{1} \Longleftrightarrow\left(\exists v_{1}, v_{2} \in V_{1}\right)\left(v_{1} \neq v_{2} \wedge \operatorname{inc}\left(v_{1}, e_{1}\right) \wedge \operatorname{inc}\left(v_{2}, e_{1}\right)\right)\right), \\
E_{1}=\operatorname{Inc}_{\mathrm{E}}\left(v_{1}\right) & :=\left(\forall e_{1}\right)\left(e_{1} \in E_{1} \Longleftrightarrow \operatorname{inc}\left(v_{1}, e_{1}\right)\right), \\
V_{1}=\operatorname{Inc}_{\mathrm{V}}\left(E_{1}\right) & :=\left(\forall v_{1}\right)\left(v_{1} \in V_{1} \Longleftrightarrow\left(\exists e_{1} \in E_{1}\right)\left(\operatorname{inc}\left(v_{1}, e_{1}\right)\right)\right) .
\end{aligned}
$$

It is easy to see that $\operatorname{Deg} 1\left(v_{1}, E_{1}\right)$ if and only if $v_{1}$ has only one neighbor in $G\left\langle E_{1}\right\rangle, \operatorname{Part}\left(V_{1}, V_{2}, V_{3}\right)$ if and only if $\left(V_{1}, V_{2}\right)$ forms a partition of $V_{3}, \operatorname{Adj}\left(v_{1}, v_{2}, E_{1}\right)$ if and only if an edge $\left\{v_{1}, v_{2}\right\}$ is in $E_{1}$, $E_{1}=\operatorname{Ind}\left(V_{1}\right)$ if and only if $E_{1}$ is the edge set of $G\left[V_{1}\right], E_{1}=\operatorname{Inc}_{\mathrm{E}}\left(v_{1}\right)$ if and only if $E_{1}$ is the set of edges between $v_{1}$ and its neighbors, and $V_{1}=\operatorname{Inc}_{\mathrm{V}}\left(E_{1}\right)$ if and only if $V_{1}$ is the vertex set of $G\left\langle E_{1}\right\rangle$. 
Using the above basic expressions, we define some expressions related to connectivity of graphs.

$$
\begin{aligned}
\operatorname{Conn}\left(E_{1}\right) & :=\left(\forall V_{2}, V_{3}\right)\left(\operatorname{Part}\left(\operatorname{Inc}_{\mathrm{V}}\left(E_{1}\right), V_{2}, V_{3}\right) \Longrightarrow\left(\exists v_{2} \in V_{2}, v_{3} \in V_{3}\right)\left(\operatorname{Adj}\left(v_{2}, v_{3}, E_{1}\right)\right)\right), \\
\operatorname{BiConn}\left(E_{1}\right): & =\left(\exists v_{1}, v_{2}, v_{3} \in \operatorname{Inc}_{\mathrm{V}}\left(E_{1}\right)\right)\left(v_{i} \neq v_{j}\right)(1 \leq i<j \leq 3) \wedge\left(\forall v_{4}\right)\left(\operatorname{Conn}\left(E_{1} \backslash \operatorname{Inc}_{\mathrm{E}}\left(v_{4}\right)\right)\right) .
\end{aligned}
$$

Clearly, $\operatorname{Conn}\left(E_{1}\right)$ if and only if $G\left\langle E_{1}\right\rangle$ is connected, and $\operatorname{BiConn}\left(E_{1}\right)$ if and only if $G\left\langle E_{1}\right\rangle$ is biconnected. Using these expressions, we can define the following expressions.

$$
\begin{aligned}
\operatorname{Forest}\left(E_{1}\right) & :=\left(\forall V_{1} \subseteq \operatorname{Inc}_{\mathrm{V}}\left(E_{1}\right)\right)\left(\neg \operatorname{BiConn}\left(\operatorname{Ind}\left(V_{1}\right) \cap E_{1}\right)\right), \\
\operatorname{Tree}\left(E_{1}\right) & :=\operatorname{Forest}\left(E_{1}\right) \wedge \operatorname{Conn}\left(E_{1}\right), \\
\operatorname{Path}\left(v_{1}, v_{2}, E_{1}\right) & :=\operatorname{Tree}\left(E_{1}\right) \wedge\left(\forall v_{3} \in \operatorname{Inc}_{\mathrm{V}}\left(E_{1}\right)\right)\left(\operatorname{Deg} 1\left(v_{3}, E_{1}\right) \Longleftrightarrow v_{3}=v_{1} \vee v_{3}=v_{2}\right) .
\end{aligned}
$$

The meanings are clear: Forest $\left(E_{1}\right)$ if and only if $G\left\langle E_{1}\right\rangle$ is a forest, Tree $\left(E_{1}\right)$ if and only if $G\left\langle E_{1}\right\rangle$ is a tree, and $\operatorname{Path}\left(v_{1}, v_{2}, E_{1}\right)$ if and only if $G\left\langle E_{1}\right\rangle$ is a $v_{1}-v_{2}$ path. Then, defining the expression $\operatorname{SpnTree}\left(E_{1}\right)$ that means $G\left\langle E_{1}\right\rangle$ is a spanning tree of $G$ is an easy task.

$$
\operatorname{SpnTree}\left(E_{1}\right):=\operatorname{Tree}\left(E_{1}\right) \wedge(\forall v)\left(v \in \operatorname{Inc}_{\mathrm{V}}\left(E_{1}\right)\right) \text {. }
$$

It is also easy to define the expression $\operatorname{Detour}\left(e_{1}, E_{1}\right)$ such that $\operatorname{Detour}\left(e_{1}, E_{1}\right)$ if and only if $G\left\langle E_{1}\right\rangle$ forms a detour for $e_{1}$ :

$$
\operatorname{Detour}\left(e_{1}, E_{1}\right):=\left(\exists v_{1}, v_{2}\right)\left(v_{1} \neq v_{2} \wedge \operatorname{inc}\left(v_{1}, e_{1}\right) \wedge \operatorname{inc}\left(v_{2}, e_{1}\right) \wedge \operatorname{Path}\left(v_{1}, v_{2}, E_{1}\right)\right) .
$$

The following expression $\operatorname{Cong}_{k}\left(e_{0}, E_{0}\right)$ means that $e_{0}$ is contained in at most $k$ detours in $G\left\langle E_{0}\right\rangle$. (Note that $e_{0}$ itself is a detour containing $e_{0}$.)

$$
\begin{aligned}
\operatorname{Cong}_{k}\left(e_{0}, E_{0}\right):=\neg\left(\exists e_{1}, \ldots, e_{k}\right)\left(\left(e_{i} \notin E_{0}\right)(1 \leq i \leq k) \wedge e_{i} \neq e_{j}(1 \leq i<j \leq k)\right. \\
\left.\wedge\left(\exists E_{i}\right)\left(e_{0} \in E_{i} \wedge E_{i} \subseteq E_{0} \wedge \operatorname{Detour}\left(e_{i}, E_{i}\right)\right)(1 \leq i \leq k)\right) .
\end{aligned}
$$

Obviously, $\operatorname{stc}(G) \leq k$ if and only if $G \vDash\left(\exists E_{0}\right)\left(\operatorname{SpnTree}\left(E_{0}\right) \wedge\left(\forall e_{0} \in E_{0}\right)\left(\operatorname{Cong}_{k}\left(e_{0}, E_{0}\right)\right)\right)$.

We can show that the treewidth of a graph of bounded degree is linear in its spanning tree congestion.

Lemma 5.2. For any connected $\operatorname{graph} G, t w(G) \leq \max \{\operatorname{stc}(G), \Delta(G)(\operatorname{stc}(G)-1) / 2\}$. Moreover, this bound is tight.

Proof. Let $k=\operatorname{stc}(G)$ and $d=\Delta(G)$. Let $T$ be a spanning tree of $G$ such that $c n g_{G}(T)=k$.

Let $T^{\prime}$ be obtained from $T$ by subdividing each edge. We use a tree decomposition with $T^{\prime}$ as tree. To each node of $T^{\prime}$, we associate the following bag. If the node is a vertex $v \in V(G)$, then put $v$ in the bag. If the node is an edge $\{v, w\} \in E(G)$ (i.e., the node is obtained by the subdivision of $\{v, w\})$, put $v$ and $w$ in the bag. Then, for every edge $\{v, w\} \notin E(T)$ select (arbitrarily) one endpoint, say $v$, and add $v$ to all bags on the path from the bag of $v$ till the bag of $w$ except the bag of $w$. This is easily seen to be a tree decomposition. 
Now, the size of a bag that corresponds to a subdivided edge $\{v, w\}$ from $T$ is at most $k+1$ : two for the $v$ and $w$, and then at most $k-1$ other edges that count for the congestion of the edge give each one vertex. Consider now a vertex $v$. Each edge not on $T$ whose detour uses $v$ as intermediate vertex counts for the congestion of two of the edges incident to $v$ in the spanning tree. For each incident edge of $v$, there are at most $k-1$ edges not on the spanning tree that count for its congestion. So, there are at most $d(k-1) / 2$ such edges. Thus, the size of a bag that corresponds to a vertex is at most $d(k-1) / 2+1$; one vertex for each edge, and then one for $v$ itself.

Observe that this bound is tight on cycles, which have degree, spanning tree congestion, and treewidth all equal to two. Furthermore, any upper bound must depend at least linearly on the spanning tree congestion. It is known that $n \times n$ grids have bounded maximum degree, treewidth $n$, and spanning tree congestion $n[16,6]$. Finally, any upper bound must also depend at least linearly on the maximum degree. Grohe and Marx [13] show that a graph family based on expanders exists in which each member has degree at most three and treewidth linear in the number of vertices of the graph.

Proposition 5.3. Let $G$ be a graph and let $G^{\prime}$ be obtained from $G$ by adding a vertex $v$ adjacent to each vertex of $G$. Then $t w(G) \leq t w\left(G^{\prime}\right) \leq t w(G)+1$ and $\operatorname{stc}\left(G^{\prime}\right) \leq \Delta(G)+1$.

Proof. By adding $v$ to each bag of a tree decomposition, $t w\left(G^{\prime}\right) \leq t w(G)+1$. As $G$ is a minor of $G^{\prime}, t w(G) \leq t w\left(G^{\prime}\right)$. A spanning tree isomorphic to $K_{1,|V(G)|}$ with $v$ at its center has congestion $\Delta(G)+1$.

Using the above proposition and the family of Grohe and Marx, we obtain a family of graphs with treewidth and maximum degree linear in the number of vertices of the graph and spanning tree congestion at most four. These facts give strong evidence for the tightness of our bound.

The upper bound improves on an earlier bound by Kozawa, Otachi, and Yamazaki [17]. Combining the above facts, we can obtain the main result of this section.

Theorem 5.4. For graphs of bounded degree, $k$-STC can be solved in linear time.

Proof. Let $G$ be a graph of bounded degree and $\Delta(G)=d$. Since $k$ and $d$ are constants, we can check whether $t w(G) \leq \max \{k, d(k-1) / 2\}$ in linear time by Bodlaender's algorithm [2]. If the output of the algorithm is "no," then $\operatorname{stc}(G)>k$ from Lemma 5.2. Otherwise, $G$ has bounded treewidth. Hence, from Theorem 5.1, we can determine whether $\operatorname{stc}(G) \leq k$ in linear time.

\section{Weighted $k$-STC is NP-complete for $k \geq 10$}

In this section, we prove the following hardness result.

Theorem 6.1. For any fixed $k \geq 10, k$-STC is NP-complete for edge weighted graphs.

Clearly, the problem belongs to NP. To show NP-completeness, we present a reduction from (3, B2)-SAT. The problem (3, B2)-SAT is a restricted version of the 3-SAT problem, which is a well-known NP-complete problem [12]. An instance $(U, C)$ of $(3, \mathrm{~B} 2)$-SAT consists of a set $U$ 
of $n$ distinct Boolean variables and a collection $C$ of $m$ clauses such that each clause has exactly three literals, and each literal occurs exactly twice. Berman, Karpinski, and Scott [1] showed the NP-completeness of (3, B2)-SAT. In their construction of a hard instance of (3, B2)-SAT, every clause has exactly three variables, that is, there is no clause like $(u, u, *),(\bar{u}, \bar{u}, *)$, or $(u, \bar{u}, *)$. Thus, in what follows, we assume that instances of $(3, \mathrm{~B} 2)$-SAT satisfy this condition as well.

The constructions in our proof are inspired by the proof of Cai and Corneil [5] for the NPcompleteness of the Weighted Tree Spanners problem. Let $k \geq 10$ be a fixed integer. For an arbitrary instance $(U, C)$ of $(3, \mathrm{~B} 2)$-SAT, we construct an edge weighted graph $G_{C}$ such that $C$ is satisfiable if and only if $\operatorname{stc}\left(G_{C}\right) \leq k$. Let $a=\lceil k / 2\rceil+1$ and $b=\lfloor k / 2\rfloor-3$. Each edge in $G_{C}$ has a weight which will be either $a, b$, or 1 . For example, if $k=10$, then the weight of an edge is six, two, or one. Clearly, the following proposition holds.

Proposition 6.2. For $k \geq 10, a+b+2=k, 2 b+6 \leq k, 2 a>k, 6 b>k$, and $4 b+4>k$.

From an instance $(U, C)$ of $(3, \mathrm{~B} 2)-\mathrm{SAT}$, the graph $G_{C}$ is constructed as follows (see Figure 1):

1. Take a vertex $x$, literal vertices $u_{i}$ and $\bar{u}_{i}$ for each variable $u_{i} \in U$, and clause vertices $c_{i}$ for each clause $c_{i} \in C$.

2. Connect $x$ to all literal vertices by literal edges of weight $b$.

3. For each variable $u_{i} \in U$, create a path of length two between $u_{i}$ and $\bar{u}_{i}$ such that edges in the path, which are called bridge edges, have weight $a$ and the center vertex of the path is a new vertex $y_{i}$.

4. For each clause $c_{i}=\left\{l_{p}, l_{q}, l_{r}\right\} \in C$, connect the clause vertex $c_{i}$ to the literal vertices $l_{p}, l_{q}$, and $l_{r}$ by clause edges of unit weight.

Clearly, the above construction can be done in polynomial time.

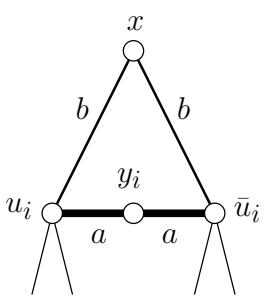

(a) Variable

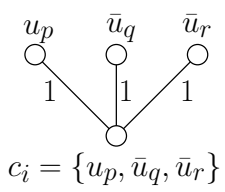

(b) Clause

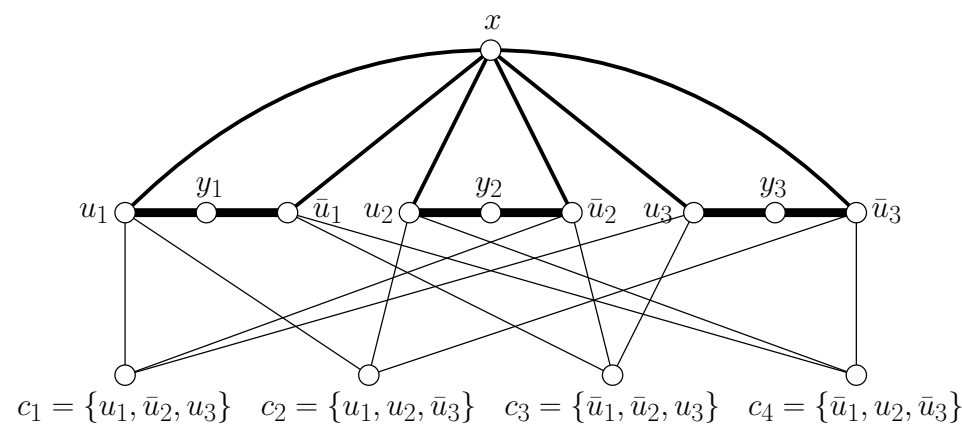

(c) $G_{C}$ with $C=\left\{\left\{u_{1}, \bar{u}_{2}, u_{3}\right\},\left\{u_{1}, u_{2}, \bar{u}_{3}\right\},\left\{\bar{u}_{1}, \bar{u}_{2}, u_{3}\right\},\left\{\bar{u}_{1}, u_{2}, \bar{u}_{3}\right\}\right\}$.

Figure 1: Gadgets, and a constructed graph.

Now, we show the following useful properties of a spanning tree of $G_{C}$ with small congestion.

Lemma 6.3. Let $T$ be a spanning tree of $G_{C}$. If $c g_{G_{C}}(T) \leq k$, then 


\section{All bridge edges are contained in $T$;}

\section{Each clause vertex is a leaf of $T$;}

3. For each variable, exactly one of its two literal edges is contained in $T$.

Proof of the first property. Since $y_{i}$ has degree two, at least one of $\left\{u_{i}, y_{i}\right\}$ and $\left\{\bar{u}_{i}, y_{i}\right\}$ must be in $T$. If $\left\{\bar{u}_{i}, y_{i}\right\}$ is not in $T$, then $\mathrm{cng}_{G_{C}, T}\left(\left\{u_{i}, y_{i}\right\}\right)=w\left(\theta\left(\left\{y_{i}\right\}\right)\right)=2 a>k$. The other case is almost the same.

Proof of the second property. Assume $T$ has the first property. By way of contradiction, suppose some clause vertex $c_{i}=\left\{l_{p}, l_{q}, l_{r}\right\}$ has degree larger than one in $T$. Let $u_{p}, u_{q}, u_{r}$ be the variables corresponding to the literals $l_{p}, l_{q}, l_{r}$, respectively. We divide the proof into two cases depending on the degree of $c_{i}$ in $T$. Recall that all bridge edges are in $T$ from the first property.

Case 1: $\operatorname{deg}_{T}\left(c_{i}\right)=3$. The three neighbors of $c_{i}$ in $T$ are $l_{p}, l_{q}$, and $l_{r}$. Let $e$ be the unique literal edge in the unique $c_{i}-x$ path in $T$. Then, $e$ separates $\{x\}$ and $\left\{u_{p}, \bar{u}_{p}, u_{q}, \bar{u}_{q}, u_{r}, \bar{u}_{r}\right\}$. Thus, $\operatorname{cng}_{G_{C}, T}(e) \geq w\left(E\left(\{x\},\left\{u_{p}, \bar{u}_{p}, u_{q}, \bar{u}_{q}, u_{r}, \bar{u}_{r}\right\}\right)\right)=6 b>k$.

Case 2: $\operatorname{deg}_{T}\left(c_{i}\right)=2$. Without loss of generality, we assume that the two neighbors of $c_{i}$ in $T$ are $l_{p}$ and $l_{q}$. Then, at most one of the literal edges of $u_{p}$ and $u_{q}$ can be in $T$. From the above case, we can assume that no clause vertex has degree three in $T$.

First, assume that none of the literal edges of $u_{p}$ and $u_{q}$ are in $T$. Let $e=\left\{x, l_{s}\right\}$ be the unique literal edge in the unique $c_{i}-x$ path in $T$. Then, $l_{s} \notin\left\{u_{p}, \bar{u}_{p}, u_{q}, \bar{u}_{q}\right\}$, and $e$ separates $\{x\}$ and $\left\{u_{p}, \bar{u}_{p}, u_{q}, \bar{u}_{q}, u_{s}, \bar{u}_{s}\right\}$. Thus, $\operatorname{cng}_{G_{C}, T}(e) \geq 6 b>k$.

Next, assume that one of the literal edges of $u_{p}$ and $u_{q}$, say $e$, is in $T$ (see Figure 2). Let us consider the clause vertices adjacent to at least one of the literal vertices $u_{p}, \bar{u}_{p}, u_{q}$, and $\bar{u}_{q}$ in $G_{C}$. If a clause vertex $c_{z}\left(\neq c_{i}\right)$ is adjacent to two vertices in $\left\{u_{p}, \bar{u}_{p}, u_{q}, \bar{u}_{q}\right\}$ in $T$, then $T$ has a cycle. Hence, if $c_{z} \neq c_{i}$ has degree two in $T$, and one of the two neighbors of $c_{z}$ is in $\left\{u_{p}, \bar{u}_{p}, u_{q}, \bar{u}_{q}\right\}$, then another neighbor, say $l_{s}$, is not in $\left\{u_{p}, \bar{u}_{p}, u_{q}, \bar{u}_{q}\right\}$. In such a case, $e$ separates $\{x\}$ and $\left\{u_{p}, \bar{u}_{p}, u_{q}, \bar{u}_{q}, u_{s}, \bar{u}_{s}\right\}$, and thus, $\operatorname{cng}_{G_{C}, T}(e) \geq 6 b>k$ (see Figure 2(a)). Therefore, every clause vertex (except for $c_{i}$ ) that has at least one of $\left\{u_{p}, \bar{u}_{p}, u_{q}, \bar{u}_{q}\right\}$ as a neighbor in $T$ is a leaf of $T$. Let $C_{1}$ be the set of such leaf clauses. Since every clause has exactly three variables, each $c \in C_{1}$ has at most two neighbors in $\left\{u_{p}, \bar{u}_{p}, u_{q}, \bar{u}_{q}\right\}$ in $G_{C}$. Hence, cng $_{G_{C}, T}(e)=w\left(\theta\left(\left\{u_{p}, \bar{u}_{p}, u_{q}, \bar{u}_{q}\right\} \cup\left\{c_{i}\right\} \cup C_{1}\right)\right) \geq 4 b+\left|C_{1}\right|+1$ (see Figure 2(b)). Since $\operatorname{cng}_{G_{C}}(T) \leq k<4 b+4$, we can conclude that $\left|C_{1}\right| \leq 2$. It is easy to see that cng $_{G_{C}, T}(e) \geq 4 b+5>k$ if $\left|C_{1}\right| \leq 2$ (see Figure 3 ).

Proof of the third property. Assume $T$ has the first and the second properties. Since $T$ is a tree and contains all bridge edges, at most one of $\left\{x, u_{i}\right\}$ and $\left\{x, \bar{u}_{i}\right\}$ can be in $T$ for each $u_{i} \in U$. Suppose $T$ contains none of them. Since any clause vertex is a leaf of $T$, there is no path between $u_{i}$ and $x$.

The next two lemmas show that $C$ is satisfiable if and only if $\operatorname{stc}\left(G_{C}\right) \leq k$, thus proving Theorem 6.1 .

Lemma 6.4. If $\operatorname{stc}\left(G_{C}\right) \leq k$ then $C$ is satisfiable. 


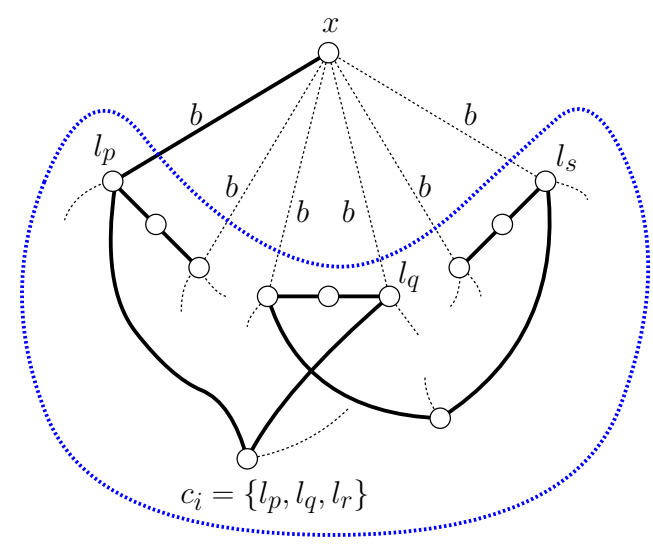

(a) Another clause vertex of degree two.

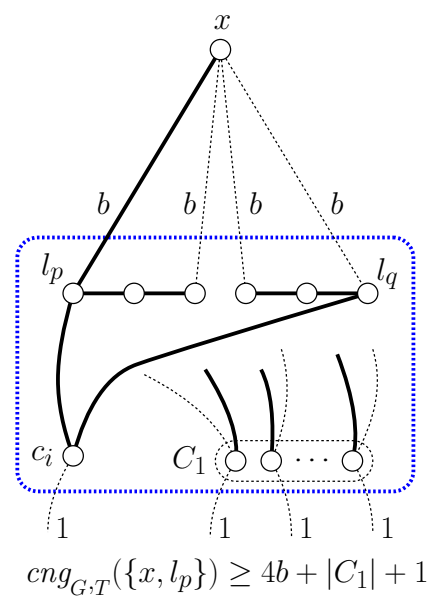

(b) No other clause vertex of degree two.

Figure 2: A clause vertex $c_{i}$ of degree two.

Proof. Let $T$ be a spanning tree of $G_{C}$ such that $c n g_{G_{C}}(T) \leq k$. From Lemma 6.3, (1) $T$ contains all bridge edges, (2) $T$ contains exactly one literal edge for each variable, and (3) every clause vertex is a leaf of $T$. From the second property, we can define a truth assignment $\xi_{T}$ by setting $\xi_{T}\left(u_{i}\right)=$ true if $\left\{x, u_{i}\right\} \in E(T)$ and $\xi_{T}\left(u_{i}\right)=$ false if $\left\{x, \bar{u}_{i}\right\} \in E(T)$. We show that $\xi_{T}$ satisfies $C$. It suffices to show that for every $c_{j} \in C$, the unique neighbor $l_{i}$ of $c_{j}$ is adjacent to $x$. If $l_{i}$ is not adjacent to $x$, then $c n g_{G_{C}, T}\left(\left\{l_{i}, y_{i}\right\}\right) \geq a+b+3>k$ (see Figure 4). This contradicts $c n g_{G_{C}}(T) \leq k$.

Lemma 6.5. If $C$ is satisfiable then $\operatorname{stc}\left(G_{C}\right) \leq k$.

Proof. Let $\xi$ be a satisfying truth assignment for $C$. We say that a literal vertex $l_{i}$ is a true vertex if $l_{i}$ becomes true by the assignment $\xi$. We construct a spanning tree $T$ of $G_{C}$ as follows:

1. Take all bridge edges.

2. Take all literal edges incident to true vertices.

3. For each clause, take an arbitrary clause edge incident with a true vertex.

Clearly, $T$ is a spanning tree of $G_{C}$. We show that $\operatorname{cng}_{G_{C}}(T) \leq k$.

Let $u_{i} \in U$. Without loss of generality, we assume that $\left\{x, u_{i}\right\} \in E(T)$. Then $T$ contains edges $\left\{x, u_{i}\right\}$ and $\left\{u_{i}, y_{i}\right\},\left\{\bar{u}_{i}, y_{i}\right\}$. From the construction of $T, T$ may contain any clause edge incident with $u_{i}$, but cannot contain any clause edge incident with $\bar{u}_{i}$. See Figure 5. Clearly, the edge $\left\{u_{i}, y_{i}\right\}$ and $\left\{\bar{u}_{i}, y_{i}\right\}$ have the same congestion, and $\operatorname{cng}_{G_{C}, T}\left(\left\{\bar{u}_{i}, y_{i}\right\}\right)=w\left(\theta\left(\left\{\bar{u}_{i}\right\}\right)\right)=a+b+2=k$. If a clause edge incident with $u_{i}$ is contained in $T$, then the edge has congestion $3 \leq k$. Obviously, $\operatorname{cng}_{G_{C}, T}\left(\left\{x, u_{i}\right\}\right)=w\left(\theta\left(\left\{u_{i}, \bar{u}_{i}\right\} \cup N_{T}\left(u_{i}\right) \backslash\{x\}\right)\right) \leq 2 b+6 \leq k$ (see Figure 5). 


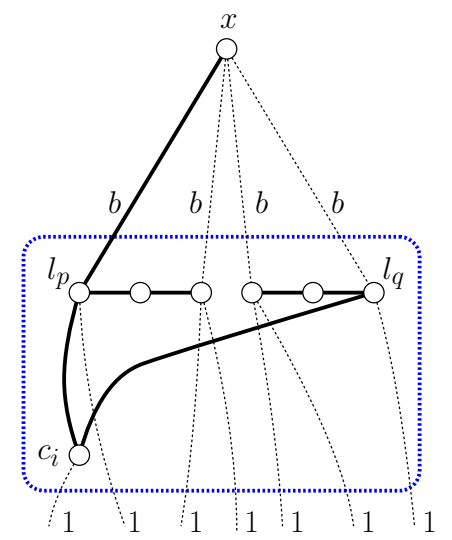

(a) $\left|C_{1}\right|=0$.

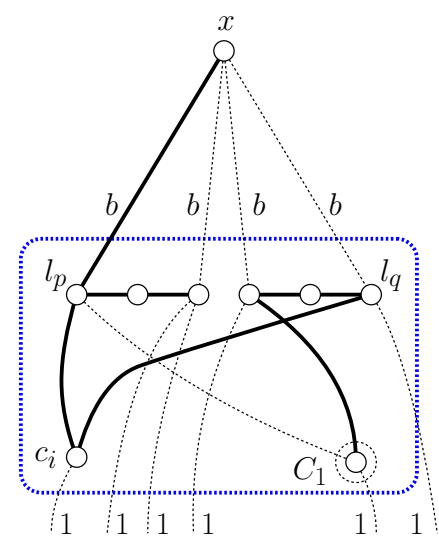

(b) $\left|C_{1}\right|=1$.

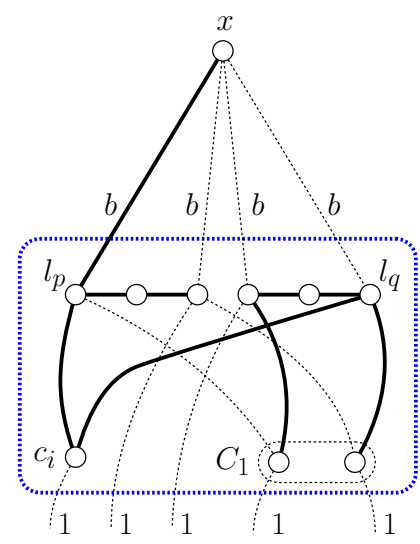

(c) $\left|C_{1}\right|=2$.

Figure 3: The cases of $\left|C_{1}\right| \leq 2$.
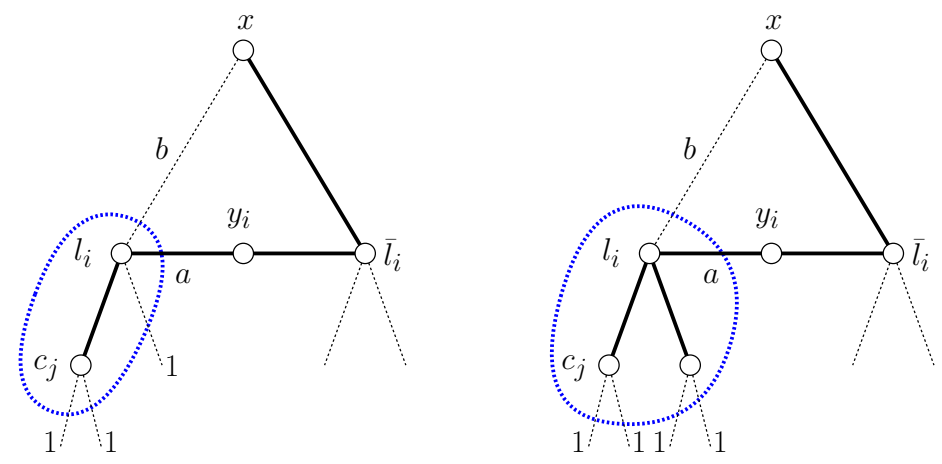

Figure 4: Unsatisfied clauses.

\section{Unweighted $k$-STC is NP-complete for $k \geq 10$}

Extending the result in the previous section, we prove the main theorem of the paper, that is, NP-completeness of $k$-STC for unweighted graphs. We need the following two lemmas.

Lemma 7.1. An edge e of weight $w \in \mathbb{Z}^{+}$can be replaced by $w$ parallel edges of unit weight without changing the spanning tree congestion.

Proof. Let $G$ be an edge weighted graph, and $e=\{u, v\} \in E(G)$ be an edge of integral weight $w \geq 2$. We denote by $G^{\prime}$ the graph obtained from $G$ by the deletion of $e$ and the addition of $w$ parallel edges $e_{1}, \ldots, e_{w}$ of unit weight between $u$ and $v$. Clearly, any spanning tree of $G^{\prime}$ contains at most one of $e_{1}, \ldots, e_{w}$. Without loss of generality, we assume for any spanning tree $T^{\prime}$ of $G^{\prime}$, $T^{\prime}$ may contain only $e_{1}$ from $\left\{e_{1}, \ldots, e_{w}\right\}$. By this assumption, we have a bijective correspondence between the spanning trees of $G$ and the spanning trees of $G^{\prime}$; we simply identify $e$ and $e_{1}$.

Let $T$ be a spanning tree of $G$, and $T^{\prime}$ the corresponding spanning tree of $G^{\prime}$. Let $P_{T}=$ $\left\{\left(A_{f}, B_{f}\right) \mid f \in E(T)\right\}$ and $P_{T^{\prime}}=\left\{\left(A_{f}, B_{f}\right) \mid f \in E\left(T^{\prime}\right)\right\}$ denote the set of the partitions of $V(G)$ 

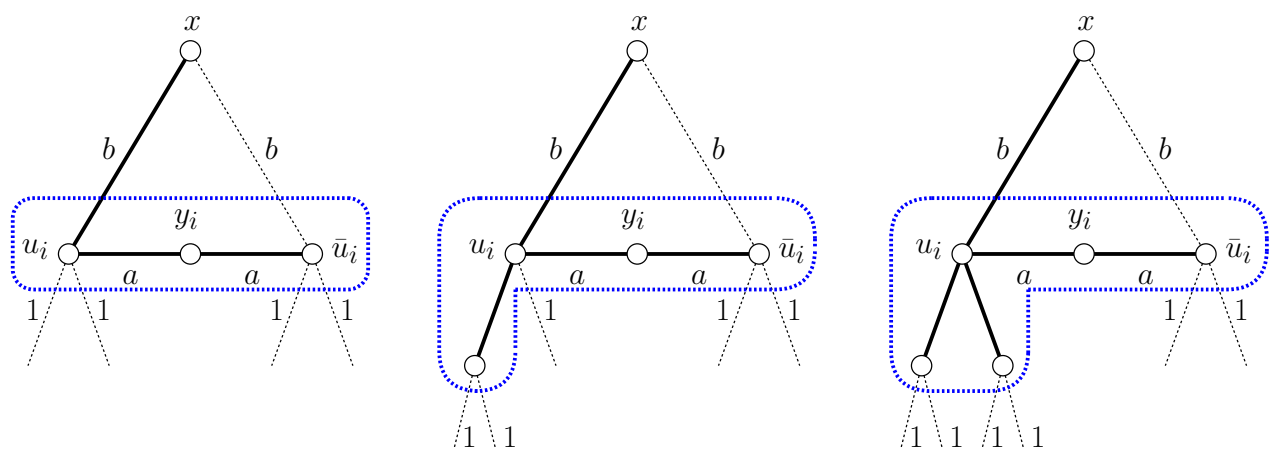

Figure 5: A spanning tree of congestion at most $k$.

defined by edges in $T$ and $T^{\prime}$, respectively. It is not difficult to see that $P_{T}=P_{T^{\prime}}$. From the definition, $c n g_{G}(T)=\max _{(A, B) \in P_{T}} w\left(E_{G}(A, B)\right)$ and $c n g_{G^{\prime}}\left(T^{\prime}\right)=\max _{(A, B) \in P_{T^{\prime}}} w\left(E_{G^{\prime}}(A, B)\right)$. If $e$ is not between $A$ and $B$, then $w\left(E_{G}(A, B)\right)=w\left(E_{G^{\prime}}(A, B)\right)$. Otherwise, $E_{G}(A, B) \backslash\{e\}=E_{G^{\prime}}(A, B) \backslash$ $\left\{e_{1}, \ldots, e_{w}\right\}$, and thus,

$$
\begin{aligned}
w\left(E_{G}(A, B)\right) & =w\left(E_{G}(A, B) \backslash\{e\}\right)+w(e)=w\left(E_{G}(A, B) \backslash\{e\}\right)+w \\
& =w\left(E_{G^{\prime}}(A, B) \backslash\left\{e_{1}, \ldots, e_{w}\right\}\right)+\left|\left\{e_{1}, \ldots, e_{w}\right\}\right|=w\left(E_{G^{\prime}}(A, B)\right) .
\end{aligned}
$$

Therefore, $\operatorname{cng}_{G}(T)=\operatorname{cng}_{G^{\prime}}\left(T^{\prime}\right)$, and hence, $\operatorname{stc}(G)=\operatorname{stc}\left(G^{\prime}\right)$.

Lemma 7.2. Edge subdivisions do not change the spanning tree congestion of unweighted graphs.

Proof. Let $G$ be a graph without edge weights, and $e=\{u, v\} \in E(G)$. We denote by $G^{\prime}$ the graph obtained from $G$ by the deletion of $e$, and the additions of a vertex $w$ and two edge $e_{1}=\{u, w\}$ and $e_{2}=\{w, v\}$. Clearly, any spanning tree of $G^{\prime}$ contains at least one of $e_{1}$ and $e_{2}$. Without loss of generality, we assume for any spanning tree $T^{\prime}$ of $G^{\prime}, e_{2} \in E\left(T^{\prime}\right)$. By this assumption, we have a bijective correspondence between the spanning trees of $G$ and the spanning trees of $G^{\prime}$; we identify $e$ and $e_{1}$, and ignore $e_{2}$.

If $\operatorname{stc}(G)=1$, then $G$ is a tree. Clearly, $G^{\prime}$ is also tree. This implies $\operatorname{stc}(G)=\operatorname{stc}\left(G^{\prime}\right)=1$. Now assume that $\operatorname{stc}(G) \geq 2$. Let $T$ be a spanning tree of $G$, and $T^{\prime}$ the corresponding spanning tree of $G^{\prime}$. Clearly, if $e_{1} \in E\left(T^{\prime}\right)$ then $\operatorname{cng}_{G^{\prime}, T^{\prime}}\left(e_{1}\right)=\operatorname{cng}_{G^{\prime}, T^{\prime}}\left(e_{2}\right)$; otherwise $\operatorname{cng}_{G^{\prime}, T^{\prime}}\left(e_{2}\right)=|\theta(\{w\})|=$ $2 \leq \operatorname{stc}(G) \leq \operatorname{cng}_{G}(T)$. It is easy to see that $c n g_{G, T}(e)=c n g_{G^{\prime}, T^{\prime}}\left(e_{1}\right)$ if $e \in E(T)$, and $c n g_{G, T}(f)=$ $c n g_{G^{\prime}, T^{\prime}}(f)$ for any $E(T) \backslash\{e\}=E\left(T^{\prime}\right) \backslash\left\{e_{1}, e_{2}\right\}$. Therefore, $c n g_{G}(T)=c n g_{G^{\prime}}\left(T^{\prime}\right)$, and hence, $\operatorname{stc}(G)=\operatorname{stc}\left(G^{\prime}\right)$.

Combining the above two lemmas, we can conclude that an edge $\{u, v\}$ of weight $w$ can be replace by $w$ internally disjoint $u-v$ paths of length two that consist of unweighted edges, without changing the spanning tree congestion. It is easy to see that this replacement can be done in $O(w)$ time. Thus, we have the following corollary.

Corollary 7.3. Let $G$ be an edge weighted graph such that the weight of every edge of $G$ is a positive integer, and the maximum weight of the edges is $w$. Then $G$ can be transformed into unweighted simple graph $G^{\prime}$ in $O(w \cdot|E(G)|)$ time, such that $\operatorname{stc}(G)=\operatorname{stc}\left(G^{\prime}\right)$. 
Now, we prove the main theorem of the paper.

Theorem 7.4. For any fixed $k \geq 10, k$-STC is NP-complete for simple unweighted graphs that have only one vertex of unbounded degree.

Proof. Let $(U, C)$ be an instance of $(3, \mathrm{~B} 2)-\mathrm{SAT}$, and $G_{C}$ the corresponding graph constructed in the previous section. From Corollary 7.3, we can construct a simple unweighted graph $G_{C}^{\prime}$ in polynomial time such that $\operatorname{stc}\left(G_{C}^{\prime}\right)=\operatorname{stc}\left(G_{C}\right)$. Clearly, $\operatorname{stc}\left(G_{C}^{\prime}\right) \leq k$ if and only if $C$ is satisfiable.

We show that the vertices other than $x$ have bounded degree. The new vertices added by subdivisions have degree two. Clause vertices have degree three in $G_{C}$. Since clause vertices are only incident to unit weight edges, they have degree three in $G_{C}^{\prime}$. Since every $y_{i}$ is incident to two bridge edges of weight $a=\lceil k / 2\rceil+1, y_{i}$ has degree $2 a \leq k+3$ in $G_{C}^{\prime}$. Literal vertex $l_{i}$ is incident to two clause edges, one bridge edge, and one literal edge that have weight one, $a$, and $b=\lfloor k / 2\rfloor-3$, respectively. Thus, $\operatorname{deg}_{G_{C}^{\prime}}\left(l_{i}\right)=a+b+2=k$. Hence, the maximum degree of $G_{C}^{\prime}$ is bounded by $k+3$, which is a constant.

\section{Concluding remarks}

We have proved that for fixed $k$, the problem of determining whether the spanning tree congestion of a given graph is at most $k$ is solvable in linear time for planar graphs, graphs of bounded treewidth, and graphs of bounded degree. We also show that the problem can be solved in linear time for any graph if $1 \leq k \leq 3$. On the other hand, we show that if the input graph has one vertex of unbounded degree, then the problem becomes NP-complete for $k \geq 10$. The complexity of $k$-STC remains open for $4 \leq k \leq 9$.

Since the problem is hard in general, an approximation algorithm with good approximation ratio is required. We say that a polynomial time algorithm for spanning tree congestion is a $c_{1^{-}}$approximation algorithm for positive number $c_{1}$ if there is a positive integer $c_{2}$ such that for any input graph $G$, the output $k$ of the algorithm satisfies $k \leq c_{1} \cdot \operatorname{stc}(G)+c_{2}$. Using NP-hardness of 10-STC, the following constant lower bound on the approximation ratio can be shown.

Theorem 8.1. There is no polynomial time $c_{1}$-approximation algorithm for the spanning tree congestion of simple unweighted graphs such that $c_{1}<11 / 10$, unless $P=N P$.

Proof. Suppose there is a polynomial time $c_{1}$-approximation algorithm $A$ for the spanning tree congestion of simple unweighted graphs with $c_{1}<11 / 10$. Let $c_{2}$ be the constant additive of $A$, that is, the output $A(G)$ of $A$ for any graph $G$ satisfies $A(G) \leq c_{1} \cdot \operatorname{stc}(G)+c_{2}$. Let $t$ be the smallest positive integer that satisfies $\left(11 / 10-c_{1}\right) \cdot t>c_{2}$.

Let $(U, C)$ be an instance of $(3, \mathrm{~B} 2)-\mathrm{SAT}$, and $G_{C}$ the corresponding graph constructed in Section 6 , where $k=10$. By $G_{C}^{\prime}$, we denote the graph obtained from $G_{C}$ by setting the edge weight as $w_{G_{C}^{\prime}}(e)=t \cdot w_{G_{C}}(e)$. Clearly, $\operatorname{stc}\left(G_{C}^{\prime}\right)=t \cdot \operatorname{stc}\left(G_{C}\right)$. Thus, if $C$ is satisfiable, then $\operatorname{stc}\left(G_{C}^{\prime}\right) \leq 10 t$; otherwise $\operatorname{stc}\left(G_{C}^{\prime}\right) \geq 11 t$. Let $G_{C}^{\prime \prime}$ be the simple unweighted graph obtained from $G_{C}^{\prime}$ by Corollary 7.3. Hence, $\operatorname{stc}\left(G_{C}^{\prime \prime}\right)=\operatorname{stc}\left(G_{C}^{\prime}\right)=t \cdot \operatorname{stc}\left(G_{C}\right)$.

Claim 8.2. $A\left(G_{C}^{\prime \prime}\right)<11 t$ if and only if $\operatorname{stc}\left(G_{C}\right) \leq 10$. 
Proof. First, assume that $A\left(G_{C}^{\prime \prime}\right)<11$. Then $t \cdot \operatorname{stc}\left(G_{C}\right)=\operatorname{stc}\left(G_{C}^{\prime \prime}\right) \leq A\left(G_{C}^{\prime \prime}\right)<11$. Thus, we have $\operatorname{stc}\left(G_{C}\right)<11$, which implies $\operatorname{stc}\left(G_{C}\right) \leq 10$. Next, assume that $\operatorname{stc}\left(G_{C}\right) \leq 10$. Then

$$
\begin{aligned}
A\left(G_{C}^{\prime \prime}\right) & \leq c_{1} \cdot \operatorname{stc}\left(G_{C}^{\prime \prime}\right)+c_{2}=c_{1} \cdot t \cdot \operatorname{stc}\left(G_{C}\right)+c_{2} \\
& =11 / 10 \cdot t \cdot \operatorname{stc}\left(G_{C}\right)-\left(11 / 10-c_{1}\right) \cdot t \cdot \operatorname{stc}\left(G_{C}\right)+c_{2} .
\end{aligned}
$$

Since $\operatorname{stc}\left(G_{C}\right) \leq 10$ and $\left(11 / 10-c_{1}\right) \cdot t>c_{2}$, we have $A\left(G_{C}^{\prime \prime}\right)<11 t-c_{2}\left(\operatorname{stc}\left(G_{C}\right)-1\right) \leq 11 t$.

From the above claim, we can use $A$ as a polynomial time algorithm for (3, B2)-SAT. As (3, B2)-SAT is NP-hard, such an algorithm cannot exist unless $\mathrm{P}=\mathrm{NP}$.

We also considered the complexity of STC or $k$-STC on some restricted graph classes. It is known that the tree spanner problem is NP-hard for chordal graphs [3] and chordal bipartite graphs [4]. It would be interesting to determine the complexity of STC or $k$-STC for these graph classes.

\section{References}

[1] P. Berman, M. Karpinski, A. D. Scott, Approximation hardness of short symmetric instances of MAX-3SAT, ECCC TR03-049 (2003).

[2] H. L. Bodlaender, A linear-time algorithm for finding tree-decompositions of small treewidth, SIAM J. Comput. 25 (1996) 1305-1317.

[3] A. Brandstädt, F. F. Dragan, H.-O. Le, V. B. Le, Tree spanners on chordal graphs: complexity and algorithms, Theoret. Comput. Sci. 310 (2004) 329-354.

[4] A. Brandstädt, F. F. Dragan, H.-O. Le, V. B. Le, R. Uehara, Tree spanners for bipartite graphs and probe interval graphs, Algorithmica 47 (2007) 27-51.

[5] L. Cai, D. G. Corneil, Tree spanners, SIAM J. Discrete Math. 8 (1995) 359-387.

[6] A. Castejón, M. I. Ostrovskii, Minimum congestion spanning trees of grids and discrete toruses, Discuss. Math. Graph Theory 29 (2009) 511-519.

[7] T. Cormen, C. E. Leiserson, R. L. Rivest, C. Stein, Introduction to Algorithms, 3rd ed., MIT Press, 2009.

[8] B. Courcelle, The monadic second-order logic of graphs III: Tree-decompositions, minor and complexity issues, Theor. Inform. Appl. 26 (1992) 257-286.

[9] R. Diestel, Graph Theory, 3rd ed., Springer-Verlag, 2005.

[10] F. F. Dragan, F. V. Fomin, P. A. Golovach, Spanners in sparse graphs, in: ICALP 2008, vol. 5125 of Lecture Notes in Comput. Sci., pp. 597-608, Springer-Verlag, 2008. 
[11] S. P. Fekete, J. Kremer, Tree spanners in planar graphs, Discrete Appl. Math. 108 (2001) 85-103.

[12] M. Garey, D. Johnson, Computers and Intractability: A Guide to the Theory of NPCompleteness, Freeman, 1979.

[13] M. Grohe, D. Marx, On tree width, bramble size, and expansion, J. Combin. Theory Ser. B 99 (2009) 218-228.

[14] P. Hliněný, S. Oum, D. Seese, G. Gottlob, Width parameters beyond tree-width and their applications, Comput. J. 51 (2008) 326-362.

[15] J. Hopcroft, R. Tarjan, Efficient planarity testing, J. ACM 21 (1974) 549-568.

[16] S. W. Hruska, On tree congestion of graphs, Discrete Math. 308 (2008) 1801-1809.

[17] K. Kozawa, Y. Otachi, K. Yamazaki, On spanning tree congestion of graphs, Discrete Math. 309 (2009) 4215-4224.

[18] H.-F. Law, Spanning tree congestion of the hypercube, Discrete Math. 309 (2009) 6644-6648.

[19] E. Lawler, Combinatorial Optimization: Networks and Matroids, Holt, Rinehart and Winston, 1976.

[20] C. Löwenstein, D. Rautenbach, F. Regen, On spanning tree congestion, Discrete Math. 309 (2009) 4653-4655.

[21] M. I. Ostrovskii, Minimal congestion trees, Discrete Math. 285 (2004) 219-226.

[22] M. I. Ostrovskii, Minimum congestion spanning trees in planar graphs, Discrete Math. 310 (2010) 1204-1209.

[23] A. Raspaud, O. Sýkora, I. Vrťo, Congestion and dilation, similarities and differences: A survey, in: 7th International Colloquium on Structural Information and Communication Complexity, SIROCCO, pp. 269-280, Carleton Scientific, 2000.

[24] N. Robertson, P. D. Seymour, Graph minors. X. Obstructions to tree-decomposition, J. Combin. Theory Ser. B 52 (1991) 153-190.

[25] S. Simonson, A variation on the min cut linear arrangement problem, Math. Syst. Theory 20 (1987) 235-252. 